\title{
Medical Image of the Week: Neuromyelitis Optica and Sarcoidosis
}

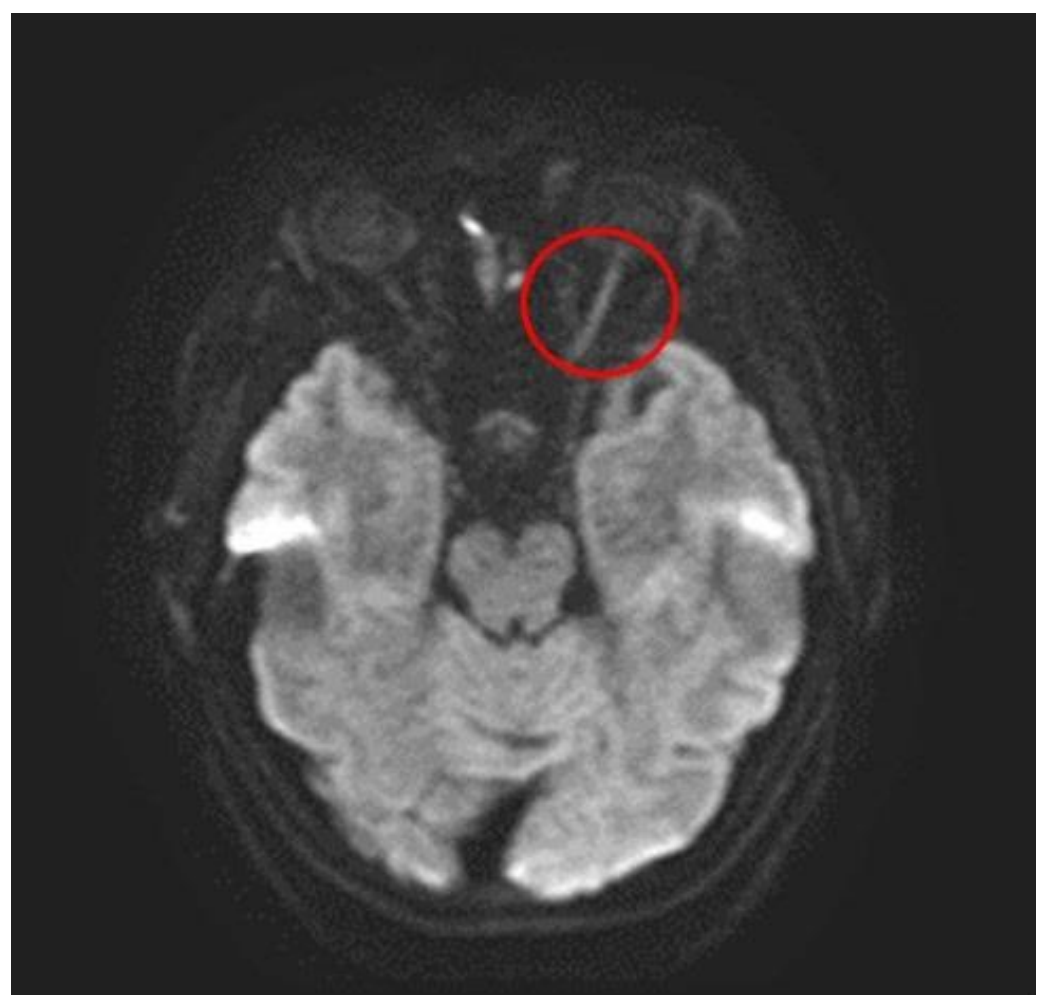

Figure 1. Magnetic resonance imaging (MRI) showing enhancement of the left optic nerve (circle) consistent with optic neuritis.

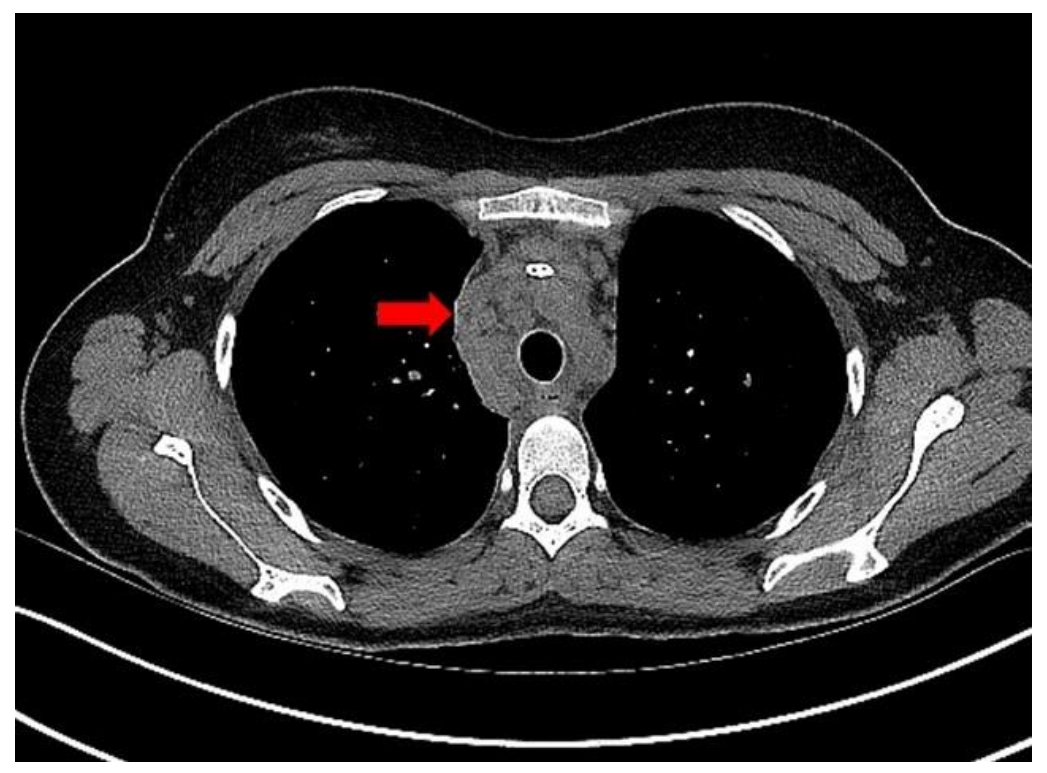

Figure 2. Thoracic CT scan showing mediastinal lymphadenopathy (arrow). 
A 33-year old woman presented to the emergency room with progressive vision loss of the left eye. A diagnosis of optic neuritis was made clinically and on magnetic resonance imaging MRI (Figure 1). With high anti-aquaporin 4 antibody titers, and compatible lesions on the MRI of the spinal cord, she was diagnosed with neuromyelitis optica (NMO). After dialysis catheter placement for plasmapheresis, an enlarged mediastinal shadow was seen. Fearing a complication of the catheter placement, a CT scan of the chest was obtained, confirming mediastinal lymphadenopathy (Figure 2).

An endobronchial ultrasound (EBUS) guided transbronchial needle aspiration of the mediastinal lymph nodes showed non-caseating granulomas, consistent with sarcoidosis in the absence of infectious work up and no known beryllium exposure.

NMO, also known as Devic's disease or Devic's syndrome, is a heterogeneous condition consisting of the inflammation and demyelination of the optic nerve (optic neuritis) and the spinal cord (myelitis). NMO and sarcoidosis can be difficult to differentiate (1). Our patient had a rarely described co-existing disease.

Tammer El-Aini MD and Bhupinder Natt MD

Division on Pulmonary, Allergy, Critical Care and Sleep

University of Arizona College of Medicine

Tucson AZ USA

\section{Reference}

1. Flanagan EP, Kaufmann TJ, Krecke KN, et al. Discriminating long myelitis of neuromyelitis optica from sarcoidosis. Ann Neurol. 2016 Mar;79(3):437-47. [CrossRef] [PubMed] 